\title{
III. The mesoscopic world
}

\section{The forces of nature}

Nature is governed by four fundamental interactions:

- The gravitational interaction which operates between objects having mass

- The electromagnetic interaction which operates between objects with electrical charge

- The strong interaction which holds nuclei together

- The weak interaction which governs beta radioactivity and other weak decays

These interactions operate over different ranges and differ greatly in strength. The main properties of these interactions are recalled in figure 21.

The gravitational interaction and electromagnetic interactions have an infinite range while the nuclear and weak interactions are of short or ultra-short range, respectively. In modern theories, the interactions between particles are considered to take place through an exchange of virtual particles (the mediators of the interaction) called gauge bosons. The mediator of the electromagnetic interaction is the photon. The strong interaction needs 8 mediators, known as gluons. In the theory, the elementary particles, the quarks, interact through gluon exchange in the same way as electric charges interact by virtual photon exchange. Three gauge bosons, the $\mathrm{W}^{+}, \mathrm{W}^{-}$and $\mathrm{Z}^{\circ}$ are the mediators of the weak interaction. For gravitation the search for the mediator, called the graviton, is still underway. In February of 2016, scientists working at the The Laser Interferometer Gravitational-Wave Observatory (LIGO) with detectors located in Hanford and in Livingston, Lousiana reported the first experimental observation of gravitational waves. This is a first step towards a possible isolation of the graviton.

\section{The role of size}

Nanoscience and nanotechnology deal with objects intermediate in size between the macroscopic world and the microscopic world. Because of the small dimensions involved, the behaviors of nanoscale objects having dimensions in the range of 1-100 $\mathrm{nm}$ can be very different from the behaviors 


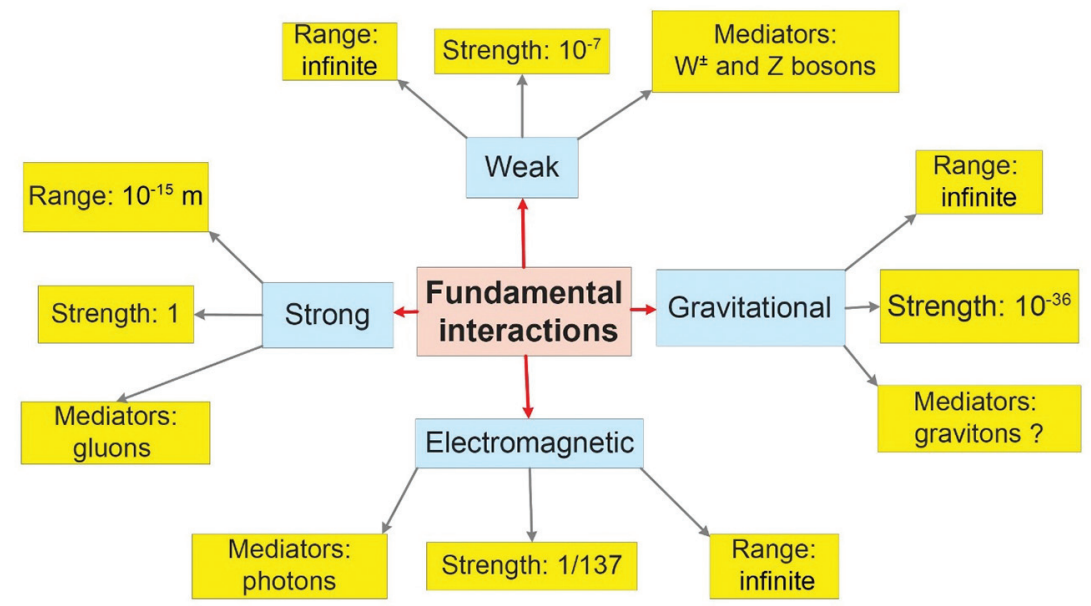

Figure 21. Main properties of the four fundamental interactions. Strengths are on a relative scale.

we have become accustomed to in the macroscopic world. Such objects can exhibit quantum behaviors.

As the size of a system decreases, the relative importance of various forces will change. Also, as the system reaches the nanoscale domain, there can be a transition from classical behavior to quantum behavior. This transition regime is typically referred to as the mesoscopic world. The transition is usually smooth and takes place over some range of sizes. This range can be wide or narrow, depending on the observable under consideration. We will call this region the quantum wall since it separates a region of sizes where classical mechanics is applicable from a region where quantum mechanics governs the physics of the observable. This is illustrated schematically in figure 22.

In this chapter we consider the role of diminishing size on various features of particular importance to nanoscale objects.

\section{Surface versus volume}

A collection of objects of small size generally has a higher surface-to-volume ratio than macroscopic objects containing the same amount of matter. If we consider, for example, a cube with a side of $1 \mathrm{~cm}$, the volume of the cube is $1 \mathrm{~cm}^{3}$ and the surface area is $6 \mathrm{~cm}^{2}$. If we cut it into nanocubes according to the scheme in figure 23, each nanocube has a volume of $1 \mathrm{~nm}^{3}$ and a surface of $6 \mathrm{~nm}^{2}$ or $6 \times 10^{-24} \mathrm{~cm}^{2}$. Since there are a lot of nanocubes, i. e. $10^{21}$, the total 


\section{Quantum wall}

Observable 1

Observable 2

Observable 3

Observable 4

Observable 5

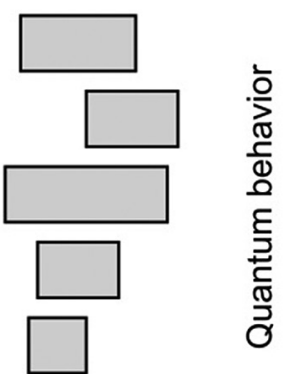

을

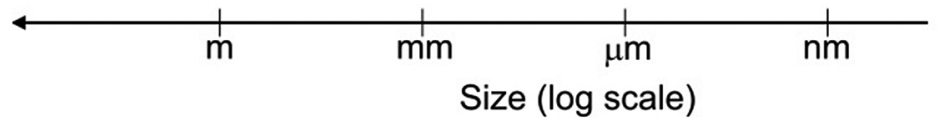

Figure 22. As the size of a system shrinks, the behavior of an observable (a variable that we can measure) can change from classical to quantum. The transition region where this happens is the quantum wall.
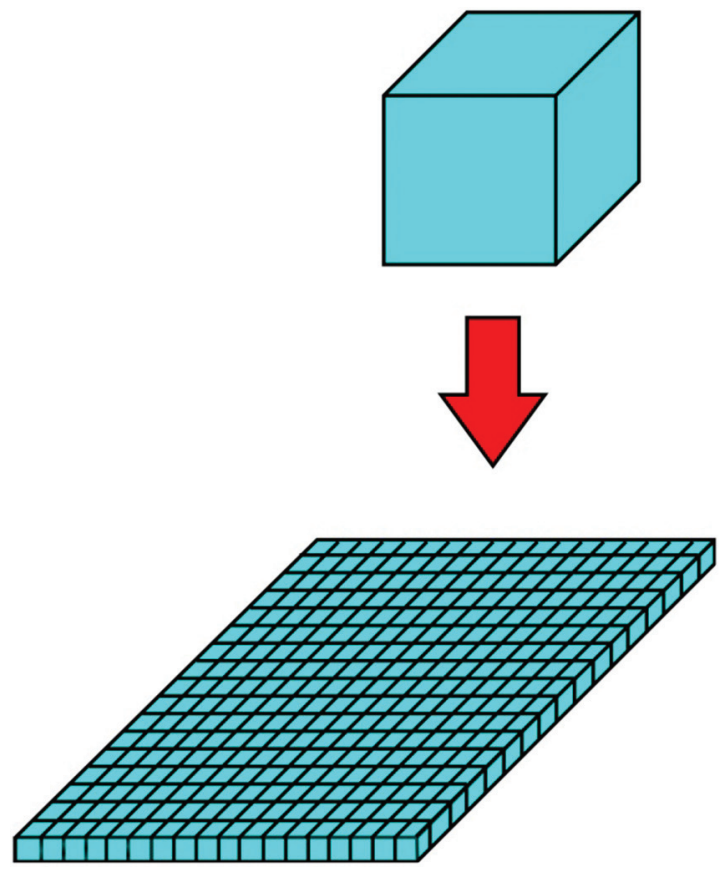

Figure 23. Dividing a cube into tiny little cubes increases substantially the surface area. See text. 
area of this set of nanocubes is $6, \mathrm{ooom}^{2}$. However the total volume of the system is the same $\left(\mathrm{cm}^{3}\right)$. The ratio of surface to volume has increased by a factor of $10^{11}$.

An increase of surface with respect to volume increases the reactivity of a material because the interface with the external environment or with other materials is larger. For example, while iron reacts with oxygen and rusts, it is difficult to burn a lump of iron. However, if this lump is ground into a small powder, or stretched into a fine thread, it burns easily. The key point to remember is that, at the nanoscale, surface effects can become dominant over volume effects.

\section{Surface tension}

For liquids, increases of surface to volume ratios can result in greatly increasing the importance of the surface tension. Surface tension finds its origin in short range intermolecular forces. Such forces are discussed in more detail in section 8 , below. For a given liquid, molecules on the surface typically have stronger attractions to molecules in the bulk of the liquid than to external molecules. In response to this difference, the liquid will tend to form drops in order to minimize the surface energy. Surface tension explains the stability of soap bubbles, the coalescence of two touching drops into a single one and why dew does not spread on flower petals.

For large volumes of liquid, surface tension is not as important as other forces which are operating. This explains why the water contained in a glass assumes the shape of the glass. Nevertheless, the surface tension exists. This explains why needles can float and small insects can easily walk on that surface. It is easy to understand that, due to surface tension, nanosized objects will have difficulties penetrating into a liquid.

\section{Nanofluids}

Unless quantum effects appear, the laws of fluid dynamics are the same, whatever the size of an object moving in a fluid. However, the fluid reacts differently depending on the size of the object. The nature of fluid flow is characterized by a parameter known as the Reynolds number. The Reynolds number is a dimensionless quantity which is the ratio of the inertial forces to the viscous forces. In the case of a body entering a fluid, the 
Reynolds number is proportional to the characteristic speed of the body and to its size and inversely proportional to the viscosity coefficient of the fluid. Two fluids with the same Reynolds number exhibit the same flow pattern. In the case of low Reynolds numbers, the flow is laminar, i. e., the liquid flows in parallel layers whereas for large Reynolds numbers it is turbulent. Viscous forces become increasingly important on the nanoscale. At the macroscopic scale, we know that turbulence provides a way to mix two different fluids together. At the nanoscale, turbulence is reduced because of the viscous forces and it is very difficult to mix two liquids using this phenomenon.

\section{Heat transfer}

Another issue at small sizes is the transfer of heat energy. Three main phenomena govern heat transfer at the macroscopic scale: conduction, convection and radiation. For nanostructures of very small size, heat transfer mechanisms are different. The classical Fourier law of heat conduction states that the rate of heat transfer through a material is proportional to the gradient in the temperature and to the area, at right angles to that gradient, through which the heat flows. On the nanoscale, this law breaks down because the mean free path of the particles becomes larger than the size of the nanostructure. The mechanism is closer to radiation than to conduction. Furthermore, at the nanoscale, because of the wave-particle duality, wave effects become important. In the mesoscopic world, where sizes intermediate between the microscopic and the macroscopic world are involved, heat transfer becomes a complicated process depending on several parameters such as the mean free path of the particles, their thermal wave length, their phase coherence, etc.

\section{Chemical bonding}

Most materials are composed of atoms of different elements bound to each other to form chemical compounds. The bonds between atoms in these compounds find their origin in the electromagnetic interaction. They vary in strength depending on their nature. In chemical compounds, the bonds between atoms range between covalent bonds, in which electron pairs are shared between two atoms, to ionic bonds, in which oppositely charged ionized atoms attract each other. The bonds are strong and not 
easy to break. Carbon-hydrogen covalent bonds have an energy of the order of $400 \mathrm{~kJ} / \mathrm{mole}$ of molecules. Ionic bonds are of comparable strengths to covalent bonds. In ionic crystals, ions located the lattice sites are bound to each other by electrostatic forces. Each ion feels the long range force from all the other ions. The net bonding is significantly larger than that between a single ion pair.

\section{Intermolecular forces}

There are also attractive interactions between atoms and molecules which are much weaker than covalent or ionic bonds, but play a major role on the properties of materials. These are generally classified as Van der Waals interactions or Van der Waals forces. As indicated in section 4, the Van der Waals forces are responsible for such phenomena as the coalescence of drops and formation of bubbles.

\section{Van der Waals forces}

The Van der Waals forces occur between atoms or molecules. They can also be present between a molecules and crystalline surfaces. They are of three types:

1. The Keesom force coming from the interaction between polar entities having permanent electric dipole moments ${ }^{5}$.

2. The Debye force occurring between entities with permanent dipole moments and those with induced dipole moments.

3. The London force coming from the interaction between entities both of which have induced dipole moments.

These 3 forces are attractive and the strengths of these forces depend on the relative distance $r$ between the two entities. The change is proportional to $1 / r^{7}$. The potential energy varies like $1 / r^{6}$. For comparison the electrostatic interaction between two charged particles varies like $1 / r^{2}$.

The Keesom force is a dipole-dipole interaction exerted between two polar molecules. The force tends to align the dipoles to increase the attraction between the species and minimize the potential energy.

The Debye force is an interaction between the permanent dipole of a polar molecule and an dipole induced in an initially non-polar molecule. 


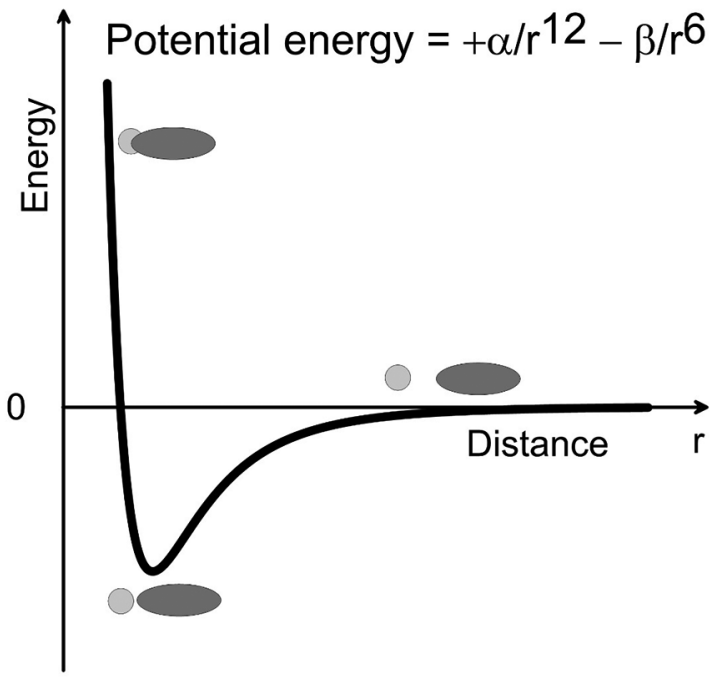

Figure 24. Schematic illustration of the potential energy curve between atomic and molecular interactions via Van der Waals forces. The repulsive core reflects the Pauli energy coming from the Pauli exclusion principle. The two parameters $\alpha$ and $\beta$ in the expression of the potential energy are determined theoretically or chosen to match experimental data.

The London forces exist between all molecules. It is the only Van de Waals interaction observed with non-polar molecules and is due to internal charge fluctuations in these molecules. In a molecule, moving electrons create an instantaneous but fluctuating dipole moment. The order of magnitude of the London forces is $\approx 2 \mathrm{~kJ} /$ mole. London forces keep the atoms of noble gases close together when they are liquefied and are responsible for the fact that benzene is a liquid at room temperature.

At very short distances the interaction between atoms and molecules turns from attractive to repulsive reflecting the fact that two identical electrons cannot be in the same quantum state ,i.e., the total wave function should be antisymmetric (the Pauli exclusion principle). This constraint on the total wave function gives rise to a short range repulsive force varying like $1 / r^{13}$ (the potential energy empirically varies like $\left.1 / r^{12}\right)$. This repulsive force is called the Pauli or Born force.

Figure 24 shows a schematic view of the potential energy of an atom and a molecule as a function of the distance $r$. This kind of potential is called a Lennard-Jones potential. As the molecules come close together the potential energy decreases because of Van der Waals interactions and then increases strongly due to the repulsive Pauli or Born interaction. 
At the nanoscale, the Van der Waals forces are much more important than gravitational forces.

\section{Hydrogen bonds}

Because of its unusual strength compared to that of other attractive Van der Waals forces, the hydrogen bond can be of particular importance. The hydrogen bond is an attractive electromagnetic interaction between a hydrogen atom already bonded to another atom in a polar molecule and a lone pair of electrons on a strongly electronegative element such as fluorine, oxygen or nitrogen. The strongest hydrogen bonds involve hydrogen-fluorine interactions. Hydrogen bonds can occur between atoms belonging to different molecules or between two atoms belonging to the same molecule.

The energy of a typical hydrogen bond is $\approx 20 \mathrm{~kJ} / \mathrm{mole}$, about an order of magnitude smaller than a strong covalent bond. The length of the bond is also about twice that of a covalent bond. Though significantly weaker than covalent bonds, hydrogen bonds can be extremely important. They are responsible for the high boiling point of water compared to other non -metallic hydrides ${ }^{6}$ and thus the fact that most of the water on the earth's surface is liquid at typical ambient temperatures. Hydrogen bonding is also the interaction which holds the double helix structure of DNA together.

\section{Semi-classical approaches}

Describing the microscopic world (atoms, molecules, nuclei...) requires us to use quantum mechanics. Quantum systems are characterized by quantum numbers which vary from small values to very large values. The quantum numbers of a system requiring a full quantum mechanical treatment are usually small because they are not too far from the ground state. In contrast, classical systems correspond to situations where the values of the quantum numbers are extremely large. As we noted above, nanotechnology covers a domain where some observables behave as classical and others behave quantum mechanically. This depends upon the nature and size of the system as well as on the external conditions. Fortunately, in many areas of nanotechnology, it is possible to meld the ideas of the classical and quantum approaches into semi-classical approaches which employ a large part of the classical thinking and invoke, ad hoc, a few quantization rules. These semi-classical approaches can then be employed to characterize nanoscale 


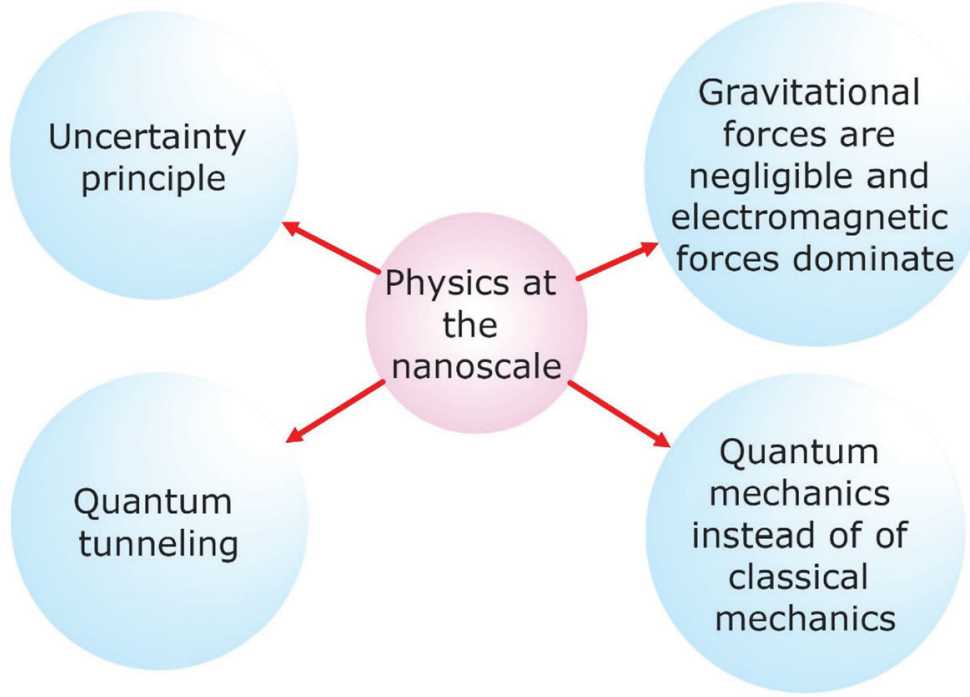

Figure 25. Important concepts manifested at the nanoscale.

phenomena. ${ }^{7}$ To the extent that a semi-classical approach captures the principal features of the phenomenon, it is useful because its application is easier than solving the full quantum problem exactly. Furthermore, because the concepts are more aligned with those of our macroscopic world, it facilitates grasping and communicating the essential features of the phenomenon.

\section{Summary}

As the dimensions of a system decrease and reach the nanometer range, new phenomena can be observed. Some of these can be understood as reflecting the natural size evolution of macroscopic behavior tempered by the relative change in importance of the forces operating on the system. In particular this is the case for surface effects which become more important than bulk effects. But there are also quantum phenomena which appear because the classical description is no longer adequate at these dimensions. In figure 25, we emphasize some concepts which play an important role in the physical and chemical behaviors of nanoscale objects. 